\title{
ON PROPERTIES OF FUNCTIONS IN EXPONENTIAL TAKAGI CLASS
}

\author{
O.E. GALKIN, S.YU. GALKINA
}

\begin{abstract}
The structure of functions in exponential Takagi class are similar to the Takagi continuous nowhere differentiable function described in 1903. These functions have one real parameter $v$ and are defined by the series $T_{v}(x)=\sum_{n=0}^{\infty} v^{n} T_{0}\left(2^{n} x\right)$, where $T_{0}(x)$ is the distance from $x \in \mathbb{R}$ to the nearest integer. For various values of $v$, we study the domain of such functions, their continuity, Hölder property, differentiability and concavity. Providing known results and proving missing facts, we give the complete description of these properties for each value of parameter $v$.
\end{abstract}

Keywords: continuity, differentiability, one-sided derivative, continuous nowhere differentiable Takagi function, Takagi class, exponential Takagi class, domain, Hölder condition, global maximum, concavity.

Mathematics Subject Classification: 26A27, 26A15, 26A16, 26A51

\section{INTORDUCTION}

Takagi function $T(x)$ was introduced by T. Takagi in 1903 in work [1], where he showed that $T(x)$ is everywhere continuous and nowhere differentiable on $\mathbb{R}$. This function can be defined by the series

$$
T(x)=\sum_{n=0}^{\infty} \frac{1}{2^{n}} T_{0}\left(2^{n} x\right), \quad x \in \mathbb{R},
$$

where $T_{0}(x)=|[x+1 / 2]-x|=|\{x+1 / 2\}-1 / 2|=\rho(x, \mathbb{Z})$ is the distance between a point $x \in \mathbb{R}$ and the nearest integer, $[x]$ is the integer part of $x,\{x\}$ is the fractional part of $x$.

Hata and Yamaguti [2, Sect. 2] replaced the sequence of the coefficients $\left\{1 / 2^{n}\right\}$ by an arbitrary sequence of coefficients $\left\{c_{n}\right\}$ in the definition of Takagi function and obtained a new family of function, which they called Takagi class.

The object of our study are real functions $T_{v}$ depending on a parameter $v$. They belong to a smaller family and are defined by the identity

$$
T_{v}(x)=\sum_{n=0}^{\infty} v^{n} T_{0}\left(2^{n} x\right), \quad x \in \mathbb{R} .
$$

We observe that as $v=0$, function $T_{v}(x)$ coincides with $T_{0}(x)$, while as $v=1 / 2$, we obtain Takagi function: $T_{1 / 2}(x)=T(x)$.

Since in the present case coefficients $c_{n}=v^{n}$ depend on $v$ by the exponential rule, the set of functions (1), where $v \in(-1 ; 1)$, will be called exponential Takagi class.

Takagi function and its generalizations are applied in various fields of mathematics, for instance, in mathematical analysis, probability theory, number theory and others. A lot of

O.E. Galkin, S.Yu. Galkina, On properties of functions in exponential Takagi Class.

(c) Galkin O.E., Galkina S.Yu. 2015.

The work is supported by RFBR (grants nos. 13-02-12155-ofi_m, 14-01-00516-a).

Submitted July 8, 2015. 
publications is devoted to these functions and their number is growing. In particular, a lot of interesting results and references were provided in surveys [3] and [4].

In the present work we study the properties of functions in the exponential Takagi class like the domain, continuity, Hölder condition, differentiability, concavity. Recalling known results and proving missing facts, we provide a complete description of these properties of functions $T_{v}$ for each value of parameter $v$. Each section of the paper is devoted to the property mentioned in its title. In the last section the provided results are illustrated graphically.

\section{DOMAIN AND CONTINUITY}

As dyadic rational numbers (or points), we shall call numbers $p / 2^{k}$, where $p \in \mathbb{Z}, k \in \mathbb{N} \cup\{0\}$.

Theorem 1. 1) If $|v|<1$, then the series (1) defining function $T_{v}(x)$ converges uniformly in $x \in \mathbb{R}$, its sum $T_{v}(x)$ is continuous and $\left|T_{v}(x)\right| \leqslant 1 /(2-2|v|)$ for each $x \in \mathbb{R}$.

2) If $|v| \geqslant 1$, then series (11) converges if and only if $x$ is dyadic rational. At that, function $T_{v}(x)$ is discontinuous on the set of dyadic rational numbers.

To prove this theorem, we shall make use of the following lemma.

Lemma 1. Let $v \neq 1 / 2, x$ be a dyadic rational point having the binary representation $x=$ $\ldots, x_{1} x_{2} \ldots x_{m}$ and $N>m$, where $m, N \in \mathbb{N}$. Then

1) If $v \in(-1 ; 1)$ and a number $h \in\left[0 ; 2^{-N}\right)$ has a binary representation $h=$ $0, \underbrace{0 \ldots 0}_{N} h_{N+1} h_{N+2} \ldots$, then the identity

$$
T_{v}(x+h)-T_{v}(x)=h \cdot\left(\frac{1-2^{N} v^{N}}{1-2 v}-2 \sum_{n=1}^{m}(2 v)^{n-1} x_{n}\right)+v^{N} T_{v}\left(2^{N} h\right)
$$

holds true.

2) The identity

$$
T_{v}\left(x+2^{-N}\right)-T_{v}(x)=2^{-N}\left(\left(1-2^{N} v^{N}\right) /(1-2 v)-2 \sum_{n=1}^{m}(2 v)^{n-1} x_{n}\right)
$$

holds true.

Proof of Lemma 1. 1) We observe first that for each number $y$ having a binary representation $0, y_{1} y_{2} \ldots$, the chain of identities

$$
T_{0}\left(0, y_{1} y_{2} \ldots\right)=T_{0}(y)=\left\{\begin{array}{cc}
y & \text { as } y_{1}=0 \\
1-y & \text { as } y_{1}=1
\end{array}=y_{1}+\left(1-2 y_{1}\right) \cdot 0, y_{1} y_{2} \ldots\right.
$$

holds true. Employing these identities, the periodicity of function $T_{0}$ and the identity $x+h=$ $\ldots, x_{1} \ldots x_{m} \underbrace{0 \ldots 0}_{N-m} h_{N+1} h_{N+2} \ldots$, we obtain

$$
\begin{aligned}
T_{v}(x+h)= & T_{v}(\ldots, x_{1} x_{2} \ldots x_{m} \underbrace{0 \ldots 0}_{N-m} h_{N+1} h_{N+2} \ldots) \\
= & \sum_{n=0}^{m-1} v^{n} T_{0}(0, x_{n+1} \ldots x_{m} \underbrace{0 \ldots 0}_{N-m} h_{N+1} \ldots) \\
& +\sum_{n=m}^{N-1} v^{n} T_{0}(0, \underbrace{0 \ldots 0}_{N-n} h_{N+1} h_{N+2} \ldots)+\sum_{n=N}^{\infty} v^{n} T_{0}\left(0, h_{n+1} h_{n+2} \ldots\right) \\
= & \sum_{n=0}^{m-1} v^{n}(x_{n+1}+\left(1-2 x_{n+1}\right) \cdot 0, x_{n+1} \ldots x_{m} \underbrace{0 \ldots 0}_{N-m} h_{N+1} h_{N+2} \ldots)
\end{aligned}
$$




$$
+\sum_{n=m}^{N-1} v^{n} \cdot 0, \underbrace{0 \ldots 0}_{N-n} h_{N+1} h_{N+2} \ldots+v^{N} T_{v}\left(2^{N} h\right) .
$$

Letting $h=0$, we find:

$$
T_{v}(x)=\sum_{n=0}^{m-1} v^{n}\left(x_{n+1}+\left(1-2 x_{n+1}\right) \cdot 0, x_{n+1} \ldots x_{m}\right) .
$$

The difference of two latter identities gives

$$
\begin{aligned}
T_{v}(x+h)-T_{v}(x)= & \sum_{n=0}^{m-1} v^{n}\left(1-2 x_{n+1}\right) \cdot 0, \underbrace{0 \ldots 0}_{N-n} h_{N+1} h_{N+2} \ldots \\
& +\sum_{n=m}^{N-1} v^{n} \cdot 0, \underbrace{0 \ldots 0}_{N-n} h_{N+1} h_{N+2} \ldots+v^{N} T_{v}\left(2^{N} h\right) \\
= & \sum_{n=0}^{N-1} v^{n} \cdot 2^{n} h-2 \sum_{n=0}^{m-1} v^{n} x_{n+1} \cdot 2^{n} h+v^{N} T_{v}\left(2^{N} h\right) \\
= & h \cdot\left(\left(1-2^{N} v^{N}\right) /(1-2 v)-2 \sum_{n=1}^{m}(2 v)^{n-1} x_{n}\right)+v^{N} T_{v}\left(2^{N} h\right) .
\end{aligned}
$$

2) If not only $x$ is dyadic rational, but $h$ is dyadic rational as well, then the series for $T_{v}(x)$ and $T_{v}(x+h)$ have only a finite amount of non-zero terms and thus, the arguments of paragraph 1$)$ are true for each $v \neq 1 / 2$. Replacing $N$ by $N-1$, we apply these arguments for the case $h=2^{-N}=0, \underbrace{0 \ldots 0}_{N-1} 1$. In view of $T_{v}(1 / 2)=1 / 2$ we obtain

$$
\begin{aligned}
T_{v}\left(x+2^{-N}\right)-T_{v}(x) & =2^{-N} \cdot\left(\frac{1-2^{N-1} v^{N-1}}{1-2 v}-2 \sum_{n=1}^{m}(2 v)^{n-1} x_{n}\right)+v^{N-1} T_{v}\left(2^{-1}\right) \\
& =2^{-N} \cdot\left(\left(1-2^{N} v^{N}\right) /(1-2 v)-2 \sum_{n=1}^{m}(2 v)^{n-1} x_{n}\right) .
\end{aligned}
$$

The proof is complete.

Proof of Theorem 1. 1) For $|v|<1$ the uniform convergence of series (11) is implied by the Weierstrass test, while the continuity of its sums is thanks to Weierstrass theorem and the continuity of its terms. The estimate $\left|T_{v}(x)\right| \leqslant 1 /(2-2|v|)$ is yielded by the inequalities

$$
\left|T_{v}(x)\right| \leqslant \sum_{n=0}^{\infty}|v|^{n}\left|T_{0}\left(2^{n} x\right)\right| \leqslant \sum_{n=0}^{\infty}|v|^{n} / 2=1 /(2-2|v|) .
$$

2) Let $|v| \geqslant 1$.

2a) We show first that if $x$ is not dyadic rational, then series (1) diverges. In this case the binary representation $x=\ldots, x_{1} x_{2} \ldots x_{n} \ldots$ is non-periodic. This is why there exists a strictly monotonically increasing sequence of indices $\left\{n_{k}\right\}_{k \in \mathbb{N}}$ such that $x_{n_{k}+1}=0$ and $x_{n_{k}+2}=1$ for each $k \in \mathbb{N}$. Therefore,

$$
T_{0}\left(2^{n_{k}} x\right)=T_{0}\left(0, x_{n_{k}+1} x_{n_{k}+2} \ldots\right)=0, x_{n_{k}+1} x_{n_{k}+2} \ldots \geqslant 0,01_{2}=1 / 4 .
$$

Hence, $\left|v^{n_{k}} T_{0}\left(2^{n_{k}} x\right)\right| \geqslant\left|v^{n_{k}}\right| / 4 \geqslant 1 / 4$, and the general terms of series (1) does not tend to zero and the series diverges. 
If $x$ is dyadic rational, then $x=p / 2^{m}$, where $p$ is integer, $m$ is non-negative integer. Therefore, as $n \geqslant m$ we have $T_{0}\left(2^{n} x\right)=T_{0}\left(2^{n-m} p\right)=0$. Thus, series (1) contains only a finite amount of non-zero terms and converges at point $x$.

$2 \mathrm{~b}$ ) Let us show that for $|v| \geqslant 1$ function $T_{v}$ is everywhere discontinuous on the set of dyadic rational points $x$. Suppose that $x$ has the binary represenation $x=\ldots, x_{1} x_{2} \ldots x_{m}$. Then by Statement 2 of Lemma 1 the identity

$$
T_{v}\left(x+2^{-N}\right)-T_{v}(x)=\left(2^{-N}-v^{N}\right) /(1-2 v)-2^{1-N} \sum_{n=1}^{m}(2 v)^{n-1} x_{n}
$$

holds true for $N>m$. Therefore, the difference $T_{v}\left(x+2^{-N}\right)-T_{v}(x)$ does not tend to zero as $N \rightarrow \infty$, and function $T_{v}$ is therefore discontinuous. The proof is complete.

Remark. 1) We note that function $T_{0}$ is even on $\mathbb{R}$, is 1-periodic and satisfies the identity $T_{0}(1-x)=T_{0}(x)$. Moreover, $T_{0}(x)=x$ as $x \in[0 ; 1 / 2]$ and $T_{0}(x)=1-x$ as $x \in[1 / 2 ; 1]$. This is why function $T_{v}$ is also even on its domain, is 1-periodic and satisfies the identity $T_{v}(1-x)=T_{v}(x)$.

2) In the case $|v|>1$ it follows from Paragraph 2b) of the proof of Theorem 1 that the difference $T_{v}\left(x+2^{-N}\right)-T_{v}(x)$ tends to infinity as $N \rightarrow \infty$ and hence, function $T_{v}$ is unbounded on any bounded interval in the set of dyadic rational numbers.

3) The simple result of Statement 1) of the theorem is provided for comparing with the result of Statement 2).

\section{LiPsCHITZ AND HÖLDER CONDITION}

3.1. As it follows from the results by K.G. Spurrier [5, Prop. 2.1.3], function $T_{v}$ satisfies Hölder condition with the exponent $\log _{2}(1 /|v|)$ on $\mathbb{R}$ for $1 / 2<|v|<1$.

3.2. It follows from the results by Shidfar and Sabetfakhri [6] that function $T_{v}$ satisfies Hölder condition with each exponent in the interval $(0 ; 1)$ on $\mathbb{R}$ for $|v|=1 / 2$.

3.3. In the case $|v|<1 / 2$ the following simple statement is true.

Theorem 2. As $|v|<1 / 2$, function $T_{v}$ satisfies Lipschitz condition on $\mathbb{R}$.

Proof. Identity (1) implies the inequality:

$$
\left|T_{v}(x)-T_{v}(y)\right| \leqslant \sum_{n=0}^{\infty}|v|^{n}\left|T_{0}\left(2^{n} x\right)-T_{0}\left(2^{n} y\right)\right| \quad \text { for each } x, y \in \mathbb{R} .
$$

By the estimate $\left|T_{0}\left(2^{n} x\right)-T_{0}\left(2^{n} y\right)\right| \leqslant\left|2^{n} x-2^{n} y\right|$ it follows that

$$
\left|T_{v}(x)-T_{v}(y)\right| \leqslant \sum_{n=0}^{\infty}|v|^{n} 2^{n}|x-y|=|x-y| /(1-2|v|) .
$$

Therefore, function $T_{v}$ satisfies Lipschitz condition.

\section{FUnCTIONAL EQUATION}

4.1. It follows from the results by de Rham [7] that for each $v \in(-1 ; 1)$ function $T_{v}$ is the unique bounded solution of the functional equation

$$
y(x)=v \cdot y(2 x)+T_{0}(x), \quad x \in \mathbb{R} .
$$

4.2. Hata and Yamaguti [8] showed that in the case $v=1 / 4$ the identity

$$
T_{v}(x)=T_{1 / 4}(x)=2\left(x-x^{2}\right) \text { as } x \in[0 ; 1]
$$

holds true. To check this identity, one can substitute function (3) into functional equation (2). 


\section{DifFERENTIABILITY}

5.1. As it has already been mentioned in Introduction, for $v=1 / 2$ function $T_{v}(x)$ coincides with Takagi function $T(x)$ and this is why it is nowhere differentiable on $\mathbb{R}$ (see [1]).

5.2. As it follows from identity (3) and the periodicity of $T_{v}(x)$, for $v=1 / 4$ this function is differentiable at all points $x \in \mathbb{R}$ except the integers.

5.3. As $v=0$, function $T_{v}$ coincides with function $T_{0}$ and hence, it is differentiable at all points $x \in \mathbb{R}$ except half-integers.

5.4. As $1 / 2 \leqslant|v|<1$, it follows from Kôno theorem [9, Thm. 2] on functions in Takagi class that function $T_{v}$ is differentiable at none of points in $\mathbb{R}$.

5.5. As $|v|<1 / 2$, the same Kôno theorem [9, Thm. 2] implies that function $T_{v}$ is differentiable almost everywhere in $\mathbb{R}$. This result can be specified as follows.

a) It follows from the results by K.G. Spurrier [5, Prop. 1.1.2] that for $|v|<1 / 2$ function $T_{v}$ is differentiable in $\mathbb{R}$ at all the points not being dyadic rational.

b) In [10, Sec. A.1.2] Mandelbrot noted without the proof that in particular, for $0<v<1 / 2$ and $v \neq 1 / 4$ function $T_{v}$ has one-sided derivatives $T_{v}^{\prime}(x \pm 0)$ at all points in $\mathbb{R}$. These derivatives are different at dyadic rational points and coincides at other points. We prove the following result being in accordance with these facts.

Theorem 3. 1) As $0<|v|<1 / 2$, at each dyadic rational point with the binary representation $x=0, x_{1} x_{2} \ldots x_{m}$ there exists one-sided derivatives $T_{v}^{\prime}(x \pm 0)=\lim _{h \rightarrow+0}\left(T_{v}(x \pm h)-T_{v}(x)\right) / h$ and the identities

$$
\begin{aligned}
& T_{v}^{\prime}(x+0)=\frac{1}{1-2 v}-2 \sum_{n=1}^{m}(2 v)^{n-1} x_{n} \\
& T_{v}^{\prime}(x-0)=\frac{1}{1-2 v}-2 \sum_{n=1}^{m}(2 v)^{n-1} x_{n}+2^{m} v^{m-1} \frac{1-4 v}{1-2 v} \quad \text { as } \quad x \notin \mathbb{Z}, \\
& T_{v}^{\prime}(x-0)=-\frac{1}{1-2 v} \quad \text { as } \quad x \in \mathbb{Z}
\end{aligned}
$$

hold true.

2) As $0<|v|<1 / 2$ and $v \neq 1 / 4$, function $T_{v}$ is not differentiable at dyadic rational points in $\mathbb{R}$.

Proof. 1) We begin with the proof of Statement 1).

1a) For each number $h \in\left(0 ; 1 / 2^{m}\right)$ one can choose natural number $N=N(h)$ such that $2^{-N-1} \leqslant h<2^{-N}$. Then employing estimate in Statement 1) of Theorem 1, we obtain:

$$
\begin{aligned}
\left|v^{N} T_{v}\left(2^{N} h\right) / h\right| & \leqslant|v|^{N} /(2(1-|v|) h)=2^{-N \log _{2} 1 /|v|} /(2 h(1-|v|)) \\
& \leqslant h^{\log _{2} 1 /|v|} /(2 h(1-|v|))=h^{\log _{2} 1 /|2 v|} /(2(1-|v|)) .
\end{aligned}
$$

Since $\log _{2} 1 /|2 v|>0$, we see that $v^{N} T_{v}\left(2^{N} h\right) / h \rightarrow 0$ as $h \rightarrow 0$. Now by Statement 1 of Lemma 1 implies identity (44).

1b) As $x \in \mathbb{Z}$, identity (6) follows identity (4) since by the periodicity and evenness we have $T_{v}^{\prime}(x-0)=T_{v}^{\prime}(-x-0)=-T_{v}^{\prime}(x+0)$.

1c) Let $x \notin \mathbb{Z}$. Without loss of generality we can assume that $x_{m}=1$ in the binary representation $x=\ldots, x_{1} x_{2} \ldots x_{m}$. Then, employing the expansion $h=0, \underbrace{0 \ldots 0}_{N} h_{N+1} h_{N+2} \ldots$, 
we obtain

$$
x-h=\ldots, x_{1} \ldots x_{m-1} 0 \underbrace{1 \ldots 1}_{N-m} \tilde{h}_{N+1} \tilde{h}_{N+2} \ldots=\tilde{x}+\tilde{h},
$$

where $\tilde{x}=\ldots, x_{1} \ldots x_{m-1} 0 \underbrace{1 \ldots 1}_{N-m}, \tilde{h}=0, \underbrace{0 \ldots 0}_{N} \tilde{h}_{N+1} \tilde{h}_{N+2} \ldots=2^{-N}-h, \tilde{h}_{n}=1-h_{n}$ as $n=N+1, N+2, \ldots$

We employ Statement 1) of Lemma 1 replacing there $x$ by $\tilde{x}, h$ by $\tilde{h}$, and $m$ by $N$ :

$$
\begin{aligned}
T_{v}(x-h)-T_{v}(\tilde{x}) & =T_{v}(\tilde{x}+\tilde{h})-T_{v}(\tilde{x}) \\
& =\tilde{h} \cdot\left(\frac{1-2^{N} v^{N}}{1-2 v}-2 \sum_{n=1}^{N}(2 v)^{n-1} \tilde{x}_{n}\right)+v^{N} T_{v}\left(2^{N} \tilde{h}\right) \\
& =\left(2^{-N}-h\right) \cdot\left(\frac{1-2^{N} v^{N}}{1-2 v}-2 \sum_{n=1}^{m-1}(2 v)^{n-1} x_{n}-2 \sum_{n=m+1}^{N-1}(2 v)^{n-1}\right)+v^{N} T_{v}\left(2^{N} h\right) .
\end{aligned}
$$

As $h=0$, this identity implies:

$$
T_{v}(x)-T_{v}(\tilde{x})=2^{-N} \cdot\left(\frac{1-2^{N} v^{N}}{1-2 v}-2 \sum_{n=1}^{m-1}(2 v)^{n-1} x_{n}-2 \sum_{n=m+1}^{N-1}(2 v)^{n-1}\right) .
$$

Deducting the former identity from the latter, we find:

$$
T_{v}(x)-T_{v}(x-h)=h \cdot\left(\frac{1-2^{N} v^{N}}{1-2 v}-2 \sum_{n=1}^{m-1}(2 v)^{n-1} x_{n}-2 \sum_{n=m+1}^{N}(2 v)^{n-1}\right)-v^{N} T_{v}\left(2^{N} h\right) .
$$

Since $x_{m}=1$, it follows that

$$
\begin{aligned}
T_{v}(x)-T_{v}(x-h)= & h \cdot\left(\frac{1-2^{N} v^{N}}{1-2 v}-\frac{2^{m+1} v^{m}-2^{N+1} v^{N}}{1-2 v}+2^{m} v^{m-1}-2 \sum_{n=1}^{m}(2 v)^{n-1} x_{n}\right) \\
& -v^{N} T_{v}\left(2^{N} h\right) .
\end{aligned}
$$

Letting $h$ to tend to zero and employing the relation $v^{N} T_{v}\left(2^{N} h\right) / h \rightarrow 0$ from Paragraph 1a), we find:

$$
T_{v}^{\prime}(x-0)=1 /(1-2 v)+2^{m} v^{m-1}(1-4 v) /(1-2 v)-2 \sum_{n=1}^{m}(2 v)^{n-1} x_{n},
$$

which is the desired identity.

2) It follows from the identities proven in Paragraph 1) that $T_{v}^{\prime}(x-0) \neq T_{v}^{\prime}(x+0)$ for $0<|v|<1 / 2, v \neq 1 / 4$ and dyadic rational $x$. This is why function $T_{v}$ is differentiable at none of dyadic rational point.

The proof is complete.

Example. By means of the formulae proven in the theorem, one can calculate, for instance, that for $v \in(0 ; 1 / 4)$, the biggest jump of the derivative at the points $x=1 / 4$ and $x=3 / 4$ is for $v=1 / 2-\sqrt{2} / 4 \approx 0,146$ and $T_{v}^{\prime}(x+0)-T_{v}^{\prime}(x-0)=6-4 \sqrt{2} \approx 0,343$, see Fig. 1 .

\section{GLOBAL MAXIMUM}

6.1. For $v=1 / 2$ the points of global and local extrema for function $T_{1 / 2}(x)=T(x)$ were found by Kahane. In particular, he proved the following statement [11]:

Theorem (Kahane, 1959). The set of the points at which Takagi function attains it global maximum equal to $4 / 3$ is the set of points having the binary representation $x=$ $\ldots, x_{1} x_{2} \ldots x_{n} \ldots$ and satisfying the condition $x_{2 k+1}+x_{2 k+2}=1$ for $k=0,1, \ldots$ 
Further results on local extrema and level sets of Takagi function can be found in surveys [3], [4] and work [14].

6.2. For $v=1 / \sqrt{2}$ it follows from the results of work [12, Lm. 5] that the global maximum of function $T_{v}$ is equal to $(2+\sqrt{2}) / 3$ and on the segment $[0 ; 1]$ is attained at two points: $1 / 3$ and $2 / 3$.

6.3. Tabor and Tabor obtained formula for the global maxima of functions $T_{v_{n}}$ on $\mathbb{R}$ for some sequence $\left\{v_{n}\right\}$. In terms of our notations their result [13, Thm. 3.1] can be formulated as follows.

Theorem (Tabor and Tabor, 2009). For each $n \in \mathbb{N}$ by $v_{n}$ we denote the unique positive solution to the equation $2 v+4 v^{2}+\ldots+2^{n} v^{n}=1$. Let $C_{v}=1 /\left(1-(4 v-1)^{\log _{2 v} v}\right)$ as $v \in(1 / 4 ; 1 / 2)$. Then

1) $v_{1}=1 / 2$, sequence $\left\{v_{n}\right\}$ decreases and converges to $1 / 4$.

2) $\max _{x \in \mathbb{R}} T_{1 / 4}(x)=1 / 2=\lim _{v \rightarrow 1 / 4+0} C_{v}$ and $\max _{x \in \mathbb{R}} T_{1 / 2}(x)=2 / 3=\lim _{v \rightarrow 1 / 2-0} C_{v}$.

3) $\max _{x \in \mathbb{R}} T_{v_{n}}(x)=C_{v_{n}}$ for $n \in \mathbb{N}, n \geqslant 2$.

6.4. For further exposition we shall need the following theorem on attaining a maximum by functions $T_{v}$ at the point $1 / 2$.

Theorem 4. 1) For $v \in[-1 / 2 ; 1 / 4]$, function $T_{v}$ has the global maximum at the point $1 / 2$ and its maximum is equal to $1 / 2$.

2) For $v \in(-1 ;-1 / 2) \cup(1 / 4 ; 1)$, function $T_{v}$ has no global maximum at the point $1 / 2$.

3) For $v \in[0 ; 1 / 4]$ and integer $n \geqslant 0$ functions

$$
S_{v, n}(x)=\sum_{k=0}^{n} v^{k} T_{0}\left(2^{k} x\right)
$$

have global maximum at the point $1 / 2$ and this global maximum is equal to $1 / 2$.

Proof. 1) We consider separately the cases $v \in[-1 / 2 ; 0)$ and $v \in[0 ; 1 / 4]$.

1a) For $v \in[-1 / 2 ; 0)$ the function $F_{v}(x)=T_{0}(x)+v T_{0}(2 x)$ is non-negative on $\mathbb{R}$. Moreover, by identity (1) defining function $T_{v}$ we obtain:

$$
T_{v}(x)=T_{0}(x)+\sum_{k=0}^{\infty} v^{2 k+1} F_{v}\left(2^{2 k+1} x\right) .
$$

Hence, since $v<0$, and $F_{v}(x) \geqslant 0$, then $T_{v}(x) \leqslant T_{0}(x) \leqslant 1 / 2$. And since $T_{v}(1 / 2)=1 / 2$, then $1 / 2$ is the point of the global maximum of function $T_{v}$.

1b) Suppose that $v \in[0 ; 1 / 4]$. If $x \in[0 ; 1]$, representation (11) of function $T_{v}$ and formula (3) yield the relations: $T_{v}(x) \leqslant T_{1 / 4}(x)=2\left(x-x^{2}\right) \leqslant 1 / 2=T_{v}(1 / 2)$. For other $x$ the inequality $T_{v}(x) \leqslant T_{v}(1 / 2)$ is true due to the periodicity of function $T_{v}$. Hence, $1 / 2$ is the point of global maximum.

2) For $v=1 / 2$ it follows from Kahane theorem (see Subsection 6.1) that $1 / 2$ is not among the points of global maximum of function $T_{v}(x)=T(x)$.

As $v \neq 1 / 2, v \in(-1 ;-1 / 2) \cup(1 / 4 ; 1)$ it is sufficient to show that for some natural $N$ the inequality $T_{v}\left(1 / 2+2^{-N}\right)>T_{v}(1 / 2)$ holds true.

By Lemma 1 for $x=1 / 2=0,1_{2}$ and $m=1$ we have:

$$
T_{v}\left(1 / 2+2^{-N}\right)=2^{-N}\left(\left(1-2^{N} v^{N}\right) /(1-2 v)-2\right)=2^{-N}\left(4 v-1-2^{N} v^{N}\right) /(1-2 v) .
$$

Therefore, for $v \in(-1 ;-1 / 2)$ the inequality $T_{v}\left(1 / 2+2^{-N}\right)>T_{v}(1 / 2)$ holds true for sufficiently large odd $N$; and for $v \in(1 / 4 ; 1), v \neq 1 / 2$ this inequality holds true for all sufficiently large $N$. 
3) As $v \in[0 ; 1 / 4]$, by the definition of function $S_{v, n}$ and Statement 1) we have: $S_{v, n}(x) \leqslant$ $T_{v}(x) \leqslant 1 / 2=S_{v, n}(1 / 2)$. Therefore, for such $v$ functions $S_{v, n}$ have the global maximum at the point $1 / 2$.

\section{Concavity on segment $[0 ; 1]$}

7.1. The following statement on concavity of functions $T_{v}$ on the segment $[0 ; 1]$ holds true.

Theorem 5. Functions $T_{v}$ are concave on the segment $[0 ; 1]$ as $v \in[0 ; 1 / 4]$ and are not concave as $v \in(-1 ; 0) \cup(1 / 4 ; 1)$.

Proof. 1) Let us show that for each $v \in[0 ; 1 / 4]$ function $T_{v}$ is concave, i.e., for each $x, y \in[0 ; 1]$ and $\alpha \in(0 ; 1)$ the inequality

$$
T_{v}(\alpha x+(1-\alpha) y) \geqslant \alpha T_{v}(x)+(1-\alpha) T_{v}(y)
$$

holds true.

Since $T_{v}(x)$ is the pointwise limit of the functions $S_{v, n}(x)=\sum_{k=0}^{n} v^{k} T_{0}\left(2^{k} x\right)$ as $n \rightarrow \infty$, in order to prove the concavity of $T_{v}$, it is sufficient to prove the concavity of functions $S_{v, n}$ for each $n=0,1,2, \ldots$. We shall do it by an induction in $n$.

As $n=0$, function $S_{v, n}(x)=T_{0}(x)$ is obviously concave on $[0 ; 1]$.

Suppose that function $S_{v, n}$ is concave on $[0 ; 1]$. Let us show that $S_{v, n+1}$ is also concave on $[0 ; 1]$, i.e., for each $x, y \in[0 ; 1]$ and $\alpha \in(0 ; 1)$ the inequality

$$
S_{v, n+1}(\alpha x+(1-\alpha) y) \geqslant \alpha S_{v, n+1}(x)+(1-\alpha) S_{v, n+1}(y)
$$

holds true.

We consider three cases: a) $0 \leqslant x \leqslant y \leqslant 1 / 2 ;$ b) $1 / 2 \leqslant x \leqslant y \leqslant 1 ;$ c) $0 \leqslant x<1 / 2<y \leqslant 1$.

a) Let $0 \leqslant x \leqslant y \leqslant 1 / 2$. Since

$$
S_{v, n+1}(x)=\sum_{k=0}^{n+1} v^{k} T_{0}\left(2^{k} x\right)=T_{0}(x)+v S_{v, n}(2 x),
$$

relation (8) is equivalent to the inequality

$$
\begin{aligned}
T_{0}(\alpha x+(1-\alpha) y)+v S_{v, n}(\alpha \cdot 2 x+(1-\alpha) \cdot 2 y) \geqslant & \alpha T_{0}(x)+(1-\alpha) T_{0}(y) \\
& +v\left(\alpha S_{v, n}(2 x)+(1-\alpha) S_{v, n}(2 y)\right) .
\end{aligned}
$$

This inequality is true by the concavity of functions $T_{0}$ and $S_{v, n}$ on the segment $[0 ; 1]$.

b) Let $1 / 2 \leqslant x \leqslant y \leqslant 1$. Then $0 \leqslant 1-y \leqslant 1-x \leqslant 1 / 2$. Replacing $x$ by $1-y, y$ by $1-x$, $\alpha$ by $1-\alpha$ in inequality (8) and employing the identity $S_{v, n+1}(t)=S_{v, n+1}(1-t)(t \in \mathbb{R})$, we obtain the desired relation.

c) We consider the last case $0 \leqslant x<1 / 2<y \leqslant 1$. Without loss of generality, we can assume that the point $\alpha x+(1-\alpha) y$ lies in the segment $[0 ; 1 / 2]$.

We choose number $\beta \in(0 ; 1)$ so that $\alpha x+(1-\alpha) y=\beta x+(1-\beta) \cdot 1 / 2$. In order to do it, we obviously should take $\beta=(1 / 2-\alpha x-(1-\alpha) y) /(1 / 2-x)$.

Since $y>1 / 2$, then $\beta<(1 / 2-\alpha x-(1-\alpha) \cdot 1 / 2) /(1 / 2-x)=\alpha$.

By the result of Paragraph a), function $S_{v, n+1}$ is concave on the segment $[0 ; 1 / 2]$. Hence,

$$
\begin{aligned}
S_{v, n+1}(\alpha x+(1-\alpha) y) & =S_{v, n+1}(\beta x+(1-\beta) \cdot 1 / 2) \\
& \geqslant \beta S_{v, n+1}(x)+(1-\beta) S_{v, n+1}(1 / 2) \\
& =S_{v, n+1}(1 / 2)-\beta\left(S_{v, n+1}(1 / 2)-S_{v, n+1}(x)\right) .
\end{aligned}
$$

In accordance with Statement 1) of Theorem 4, as $v \in[0 ; 1 / 4]$, the inequalities $S_{v, n+1}(x) \leqslant$ $S_{v, n+1}(1 / 2)$ and $S_{v, n+1}(y) \leqslant S_{v, n+1}(1 / 2)$ hold true. In view of these inequalities and $\beta<\alpha$, we get the concavity for function $S_{v, n+1}$ :

$$
S_{v, n+1}(\alpha x+(1-\alpha) y) \geqslant S_{v, n+1}(1 / 2)-\alpha\left(S_{v, n+1}(1 / 2)-S_{v, n+1}(x)\right)
$$




$$
=\alpha S_{v, n+1}(x)+(1-\alpha) S_{v, n+1}(1 / 2) \geqslant \alpha S_{v, n+1}(x)+(1-\alpha) S_{v, n+1}(y) .
$$

2) As $v \in(-1 ; 0)$, function $T_{v}$ is not concave on the segment $[0 ; 1]$ since

$$
T_{v}(1 / 4)=T_{0}(1 / 4)+v T_{0}(1 / 2)=1 / 4+v / 2<1 / 4 \text {. }
$$

Therefore, as $x=0, y=1 / 2, \alpha=1 / 2$, concavity condition (7) fails.

3) It remains to show that as $v \in(1 / 4 ; 1)$, function $T_{v}$ is not concave on the segment $[0 ; 1]$. Since in accordance with Statement 2) of Theorem 4 there is no global maximum at the point $1 / 2$, for some $x_{0} \in[0 ; 1]$ we have $T_{v}\left(x_{0}\right)>T_{v}(1 / 2)$. Then $T_{v}\left(1-x_{0}\right)=T_{v}\left(x_{0}\right)>T_{v}(1 / 2)$ and as $x=x_{0}, y=1-x_{0}, \alpha=1 / 2$, concavity condition (7) fails.

The proof is complete.

7.2. We also mention the result of Tabor and Tabor [13, Corollary 2.1] who proved that for $v \in[1 / 4,1 / 2]$ functions $T_{v}$ are $\left(1, \log _{2}(1 / v)\right)$-semi-convex, i.e., they satisfy the inequalities

$$
T_{v}\left(\frac{x+y}{2}\right) \leqslant \frac{T_{v}(x)+T_{v}(y)}{2}+|x-y|^{\log _{2}(1 / v)} ; \quad x, y \in \mathbb{R} .
$$

8. Illustration of PRESENTED REsults

Here we illustrate the results of the work by the example of functions $T_{v}$ for $v=0,146$ and $v=-0,64$.

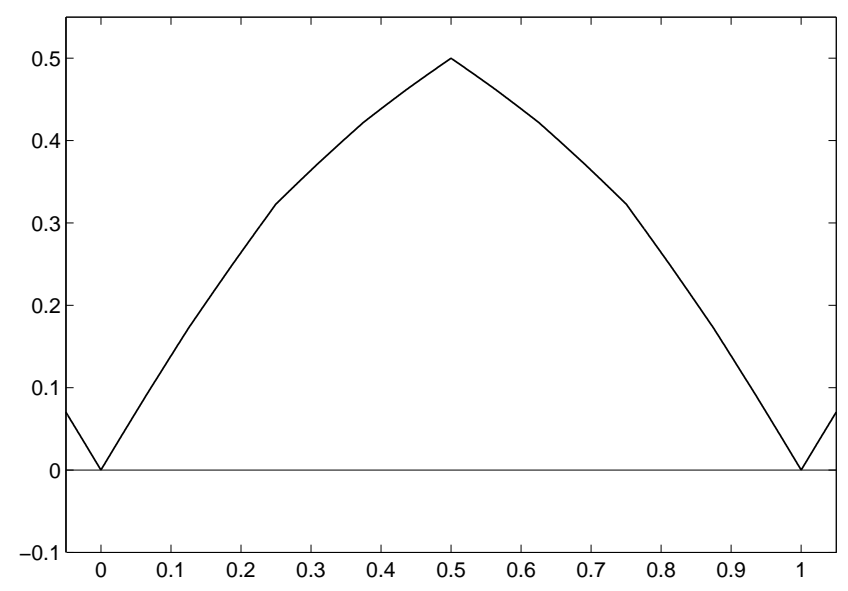

FiguRE 1.

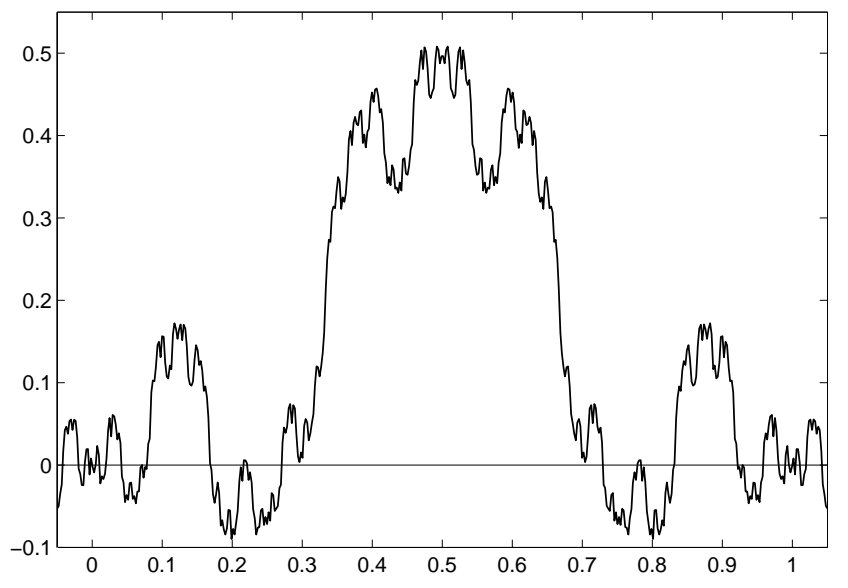

FiguRE 2.

Function $y=T_{0,146}(x)$ (its graph is given in Fig. 1) satisfies Lipschitz condition in $\mathbb{R}$ (see Theorem 2). It is not differentiable at dyadic rational points and is differentiable at other points (see Subsection 5.5). It has a global maximum $1 / 2$ at point $1 / 2$ (see Theorem 4). It is concave on segment $[0 ; 1]$ (see Theorem 51).

Function $y=T_{-0,64}(x)$ (see its graph in Fig. 2) satisfies Hölder condition with the exponent $\log _{2}(25 / 16) \approx 0,644$ in $\mathbb{R}$ (see Subsection 3.1). It is differentiable at none of the points in $\mathbb{R}$ (see Subsection 5.4). It has no global maximum at point $1 / 2$ (see Theorem 44). It is not concave on the segment $[0 ; 1]$ (see Theorem 51).

\section{BIBLIOGRAPHY}

1. T. Takagi. A simple example of a continuous function without derivative // Proc. Phys. Math. Soc. Japan. 1, 176-177 (1903).

2. M. Hata, M. Yamaguti. Takagi function and its generalization // Japan J. Appl. Math. 1:1, 183-199 (1984).

3. P.C. Allaart, K. Kawamura. The Takagi function: a survey // Real Anal. Exchange. 37:1, 1-54 $(2011 / 12)$. 
4. J.C. Lagarias. The Takagi Function and Its Properties // In RIMS Kokyuroku Bessatsu: Functions and Number Theory and Their Probabilistic Aspects. Kyoto. 34, 153-189 (2012).

5. K.G. Spurrier. Continuous Nowhere Differentiable Functions. Senior Thesis. South Carolina Honors College, South Carolina (2004).

6. A. Shidfar, K. Sabetfakhri. On the Continuity of Van Der Waerden's Function in the Hölder Sense // Amer. Math. Monthly. 93:5, 375-376 (1986).

7. de Rham G. Sur un exemple de fonction continue sans dérivée // Enseign. Math. II. Sér 3. 71-72 (1957).

8. M. Hata, M. Yamaguti. Weierstrass's function and chaos // Hokkaido Math. J. 12, 333-342 (1983).

9. N. Kôno. On generalized Takagi functions // Acta Math. Hungar. 49, 315-324 (1987).

10. B.B. Mandelbrot. Fractal landscapes without creases and with rivers // In The Science of Fractal Images, eds. H.-O. Peitgen, D. Saupe, Springer-Verlag, New-York, 247 (1988).

11. J.P. Kahane. Sur l'exemple, donné par M. de Rham, d'une fonction continue sans dérivée // Enseignement Math. 5, 53-57 (1959).

12. S.Yu. Galkina. On the Fourier-Haar coefficients of functions of bounded variation // Matem. Zametki. 51:1, 42-54 (1992). [Math. Notes. 51:1, 27-36 (1992).]

13. J. Tabor, J. Tabor. Takagi functions and approximate midconvexity // J. Math. Anal. Appl. 356:2, 729-737 (2009).

14. P.C. Allaart. On the level sets of the Takagi-van der Waerden functions // J. Math. Anal. Appl. 419:2, 1168-1180 (2014).

Galkin Oleg Evgen'evich,

Institute of information technology, mathematics and mechanics NNSU,

Gagarin av., 23,

603950, Nizhni Novgorod, Russia

E-mail: olegegalkin@ya.ru

Galkina Svetlana Yur'evna,

Institute of information technology, mathematics and mechanics NNSU,

Gagarin av., 23,

603950, Nizhni Novgorod, Russia

E-mail: svetlana.u.galkina@mail.ru 